\title{
AVALIAÇÃO DE INSETICIDAS NO CONTROLE DE Sitophilus oryzae (LINNAEUS) (COLEOPTERA, CURCULIONIDAE), E Rhyzopertha dominica (FABRICIUS) (COLEOPTERA, BOSTRICHIDAE) EM CEVADA.
}

\author{
Insecticide Evaluation in the Control of Sitophilus oryzae \\ (Linnaeus) (Coleoptera, Curculionidae), and Rhyzopertha \\ dominica (Fabricius) (Coleoptera, Bostrichidae) in Barley.
}

\author{
Rui S. Furitatti ${ }^{1}$ \\ Airton Rodrigues Pinto Junior ${ }^{2}$ \\ Paulo R. V. da S. Pereira ${ }^{3}$
}

\section{Resumo}

Foi determinada a eficiência de inseticidas no controle de Sitophilus oryzae (Linnaeus) (Coleoptera, Curculionidae) e Rhyzopertha dominica (Fabricius) (Coleoptera, Bostrichidae) em cevada, expondo-os a grãos tratados em laboratório. Cada parcela, composta por $1,2 \mathrm{~kg}$ de cevada, foi tratada com $5 \mathrm{ml}$ da concentração/kg de cevada, usando um pulverizador propulsionado a ar. Quinze dias após o tratamento, foi retida uma amostra de $50 \mathrm{~g}$ de grãos de cada parcela, colocadas em frascos plásticos com 10 adultos de cada espécie. Repetiu-se essa prática a cada 30 dias até 165 dias após o tratamento. 0 número de insetos mortos foi contado 15 dias após cada infestação, sendo as avaliações realizadas aos 30, 60, 90, 120, 150 e 180 dias após a aplicação dos inseticidas. Fenitrotiom $\left(10 \mathrm{~g}\right.$ i.a.t $\left.\mathrm{t}^{1}\right)$, deltametrina $\left(0,375 \mathrm{~g}\right.$ i.a. $\left.\mathrm{t}^{1}\right)$ e fenitrotiom + esfenvalerate $\left(7,5+0,375 ; 10+0,5\right.$ e 12,5 +0,625 g i.a.t $\left.t^{1}\right)$ foram efetivos no controle de S. oryzae até 180 dias. Deltametrina $\left(0,375\right.$ g i.a.t $\left.t^{1}\right)$ e fenitrotiom + esfenvalerate $\left(10+0,5\right.$ e $12,5+0,625$ g i.a. $\left.\mathrm{t}^{1}\right)$ foram efetivos no controle de R. dominica até 180 dias. Fenitrotiom + esfenvalerate $\left(7,5+0,375 \mathrm{~g}\right.$ i.a.t $\left.{ }^{1}\right)$ foram efetivos até 120 dias. $\mathrm{O}$ uso de misturas de organofosforados mais piretróides foram mais eficazes no controle de S. oryzae e R. dominica, quando comparados com esses inseticidas usados individualmente.

Palavras-chave: Insecta; Controle químico; Pragas de produtos armazenados.

1 Prof. Dr. lotado no Departamento de Fitotecnia e Fitossanidade, UEPG, Praça Santos Andrade, s/n, 84010-330, Ponta Grossa, PR, e-mail: furiatti@convoy.com.br . Caixa postal 992/993. CEP : 84010-970 Rui S. Furiatti.

2 Prof. Dr. Adjunto III lotado na Pontifícia Universidade Católica do Paraná, Centro de Ciências Agrárias e Ambientais, BR 376, km 14, São José dos Pinhais, Caixa postal 129, 83010-500, PR, e-mail: airton.junior@pucpr.br

3 Pesquisador Dr. lotado na Embrapa Roraima, BR 174, km 8, Distrito Industrial, Boa Vista, Caixa postal 133, 69301-970, RR, e-mail: paulo@cpafrr.embrapa.br 


\section{Abstract}

Efficacy of insecticides to control Sitophilus oryzae (Linnaeus) (Coleoptera, Curculionidae) and Rhyzopertha dominica (Fabricius) (Coleoptera, Bostrichidae) in barley was evaluated. The efficiency of insecticides against Sitophilus oryzae (Linnaeus, 1763) (Coleoptera, Curculionidae) and Rhyzopertha dominica (Fabricius, 1792) (Coleoptera, Bostrichidae) was determined exposing them to treated barley in laboratory. Each plot, which was composed by $1.2 \mathrm{~kg}$ of barley, was treated with $5 \mathrm{ml}$ of the concentration/ $\mathrm{kg}$ of barley using a propelling air atomizer. Fifteen days after treatment a sample of $50 \mathrm{~g}$ of barley grains was taken, from each plot, placed in plastic bottle with 10 adult insects of each species. This was repeated every 30 days until 165 days after treatment. The number of dead insects was counted 15 days after each infestation, thus the evaluations were made at 30, 60, 90, 120, 150 and 180 days after the insecticide application. Fenitrothion $(10 \mathrm{~g}$ a.i./ $/ \mathrm{t})$, deltamethrin $(0.375 \mathrm{~g}$ a.i./t) and fenitrothion + esfenvalerate $(7.5+0.375 ; 10+0.5$ and $12.5+0.625 \mathrm{~g}$ a.i./ $\mathrm{t})$ were effective against $\mathrm{S}$. oryzae until 180 days. Deltamethrin $(0.375 \mathrm{~g}$ a.i./ $/ \mathrm{t})$ and fenitrothion + esfenvalerate $(10+0.5$ and $12.5+0.625 \mathrm{~g}$ a.i. $/ \mathrm{t})$ were effective against R dominica until 180 days. Fenitrothion + esfenvalerate $(7.5+0.375 \mathrm{~g}$ a.i./t $)$ was effective until 120 days. The use of mixtures of organophosphorous plus pyretroids were more effective against S. oryzae and $\mathrm{R}$. dominica when compared with these insecticides used alone.

Keywords: Insecta; Chemical control; Stored-product pests.

\section{Introdução}

A FAO estima que as perdas quantitativas anuais causadas por pragas durante o período de armazenamento de grãos são da ordem de $10 \%$ da produção mundial. Esta estimativa refere-se, apenas, ao ataque dos insetos ao embrião e ao endosperma das sementes, não entrando em consideração o aquecimento da massa de grãos provocada pela atividade dos insetos, conseqüente ataque de fungos e também a diminuição do valor nutritivo dos grãos (ALMEIDA, 1989).

No Brasil, em função das precárias condições de armazenamento no meio rural e de condições climáticas favoráveis ao crescimento da população de pragas, as perdas atingem cerca de $10 \%$ da produção total de grãos (VENDRAMIN et al., 1988). A baixa disponibilidade de inseticidas registrados para o controle de pragas de produtos armazenados contribui para o agravamento deste problema, uma vez que nem todos os produtos podem ser utilizados para todas as pragas e nas diversas situações de armazenamento encontradas no Brasil (LORINI; SCHNEIDER, 1994).

Na desinfestação prévia dos focos residuais de pragas em armazéns, paióis, tulhas, silos e graneleiros, recomenda-se à pulverização de pisos paredes e outras partes acessíveis destes depósitos. O teor de umidade dos grãos a serem armazenados não deve exceder a determinados limites (ao redor de 12 a 13\% para cereais e de 11 a $12 \%$ para soja e feijão).

No Brasil, Pacheco, Sartori e Yokomizo (1988) encontraram linhagens de S. oryzae insensíveis ao inseticida deltamethrina, mesmo com uma dose de $2 \mathrm{mg} / \mathrm{kg}$ de trigo.
Yadav (1988), trabalhando com trigo armazenado em saco de juta, demonstrou que a toxicidade da deltamethrina para S. oryzae e R dominica persistiu por 120 a 170 dias na superfície da juta, e a proteção contra os mesmos insetos durou por 6 meses de armazenagem.

Schiffers et al. (1989) constataram que a deltamethrina foi o inseticida mais eficaz no controle de R. dominica e S. oryzae em trigo.

Bareth e Gupta (1989a), trabalhando com $\mathrm{R}$. dominica em trigo, mostraram que a deltamethrina plus a 0,015\% e deltamethrina a 0,075 ppm foram os produtos mais eficientes no controle deste inseto.

Bareth e Gupta (1989b) testaram 6 inseticidas no controle de R. dominica, concluindo que a deltamethrina a 2 ppm mostrou a melhor eficácia contra este inseto, seguido por fenitrothion a $20 \mathrm{ppm}$. Demonstraram também que nenhum dos inseticidas alterou a germinação das sementes.

Suchita etal. (1989), baseando-se na CL50 do S. oryzae, concluíram que o fenpropathrim foi o produto que mostrou o menor controle, quando comparado com cipermethrin, malathion, fenvalerate e flucitrinate.

Islam et al. (1989), comparando deltamethrina com carbaril contra S. oryzae, concluíram que a deltamethrina foi mais efetiva por um período de nove meses em trigo armazenado.

Giga e Zvoutete (1990), trabalhando com milho infestado com S. zeamais, observaram que o fenitrothion e a deltamethrina foram eficientes até 120 e 90 dias após o tratamento, respectivamente. 
Duguet e Quan (1990) demonstraram que a deltamethrina $(0,5 \mathrm{ppm}+5,0 \mathrm{ppm}$ de butóxido de piperonila, BPO) protegeu o arroz contra $R$. dominica, S. zeamais e S. oryzae em trabalho realizado na China.

A aplicação de deltamethrina a $1 \mathrm{mg} / \mathrm{kg}$, com BPO a $8 \mathrm{mg} / \mathrm{kg}$, controlou as progênies de S. zeamais e com BPO a $16 \mathrm{mg} / \mathrm{kg}$ chegou-se a 48 semanas de proteção. Entretanto, papéis impregnados com deltamethrina sinergizada foram menos efetivos contra biótipos de S. zeamais resistentes a deltamethrina e organofosforados (SAMSON; PARKER; HALI, 1990).

Guedes e Silva (1992) obtiveram bom controle de S. zeamais com a deltamethrina $(0,43$ ppm) em milho armazenado, após 30, 60, 90, 120, 150 e 180 dias do tratamento.

\section{Material e métodos}

O experimento foi realizado no Departamento de Zoologia da Universidade Federal do Paraná, onde foram mantidas em sala climatizada $\left(25 \pm 2^{\circ} \mathrm{C}\right)$ as colônias de S. oryzae e de $\mathrm{R}$. dominica, provenientes de diversos pontos do Estado do Paraná usadas neste experimento. O delineamento estatístico utilizado foi de blocos casualizados, com quatro repetições.

Cada parcela foi constituída de $1,3 \mathrm{~kg}$ de trigo, que, depois de tratados, foram acondicionados em sacos de ráfia e armazenados em sala climatizada $\left(25 \pm 2^{\circ} \mathrm{C}\right)$.

A aplicação dos inseticidas foi efetuada com um pulverizador manual propulsionado a ar, equipado com bico pneumático com vazão de 5,0 l/t. Cada parcela recebeu $5 \mathrm{ml}$ de caldo por kg de grãos de cevada e na testemunha apenas água. Os inseticidas foram aplicados dentro de sacos plásticos de 0,4 x 0,5 m e homogeneizados por dois minutos.

Nos tratamentos utilizou-se: 0 inseticida organofosforado, fenitrothion (Sumigran ${ }^{\circledR} 500$ CE, na dosagem de 10 ml.ton ${ }^{-1}$.); o piretróide, deltametrina (k-obiol ${ }^{\circledR}$ 2,5 CE na dosagem de 15 ml.ton $^{-1}$.) e a mistura pronta de um organofosforado + um piretróide, fenitrothion + esfen- valerate (Sumigranplus ${ }^{\circledR}$, na dosagem de 15, 20

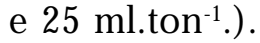

Para a avaliação da eficiência, em intervalos de 15 dias após a aplicação dos inseticidas, $50 \mathrm{~g}$ de cada parcela, foram infestadas com 10 insetos adultos, não sexados e sem padronização de idade das espécies testadas, acondicionadas em copos plásticos de 0,5 l em sala climatizada $\left(25 \pm 2{ }^{\circ} \mathrm{C}\right)$. A avaliação do número de insetos mortos foi feita 15 dias após cada infestação, ou seja, aos 30, 60, 90, 120, 150 e 180 dias após a aplicação dos inseticidas.

Considerou-se eficiente o inseticida que não apresentou diferença estatística a $100 \%$ de mortalidade de S. oryzae e R. dominica, e diferença estatística significativa da testemunha.

O número de insetos mortos por parcela foi submetido à análise de variância e as diferenças entre as médias discriminadas pelo teste de Tukey a 5\%. A eficiência relativa dos inseticidas foi calculada pela fórmula de Abbot (ABBOTT, 1925).

\section{Resultados e discussão}

Todos os tratamentos, em todas as avaliações, foram estatisticamente diferentes da testemunha, para R. dominica e para S. oryzae.

Para S. oryzae, todos os tratamentos foram eficazes até os 180 dias após a sua aplicação nos grãos de cevada. Apenas o tratamento 5 reduziu a eficácia ao chegar nesta dada, porém mantendo-se eficaz. Contra este inseto não se observou diferença significativa entre os tratamentos em nenhuma das amostragens.

No que se refere a R. dominica, todos os tratamentos foram considerados eficazes até os 120 dias após a sua aplicação, excetuando-se o tratamento 4, o qual não se mostrou eficaz em nenhuma das amostragens. Estes resultados se modificaram aos 150 dias, quando o tratamento 1 perdeu a eficácia e o tratamento 5 a teve reduzida. Aos 180 dias, a situação registrada na avaliação anterior permaneceu inalterada, observando-se, ainda, uma queda maior na eficácia do tratamento 5 . 


\section{Tabela 1 - Número médio de adultos mortos $(\mathrm{X})$ de Sitophilus oryzae e porcentagem de} eficácia dos inseticidas (E\%) em diferentes períodos de infestação após o tratamento dos grãos de cevada.

Table 1 - Average number of dead adults (x) of Sitophilus oryzae and percentage of insecticides effectiveness (E\%) in different periods of infestation after the treatment of the barley grains.

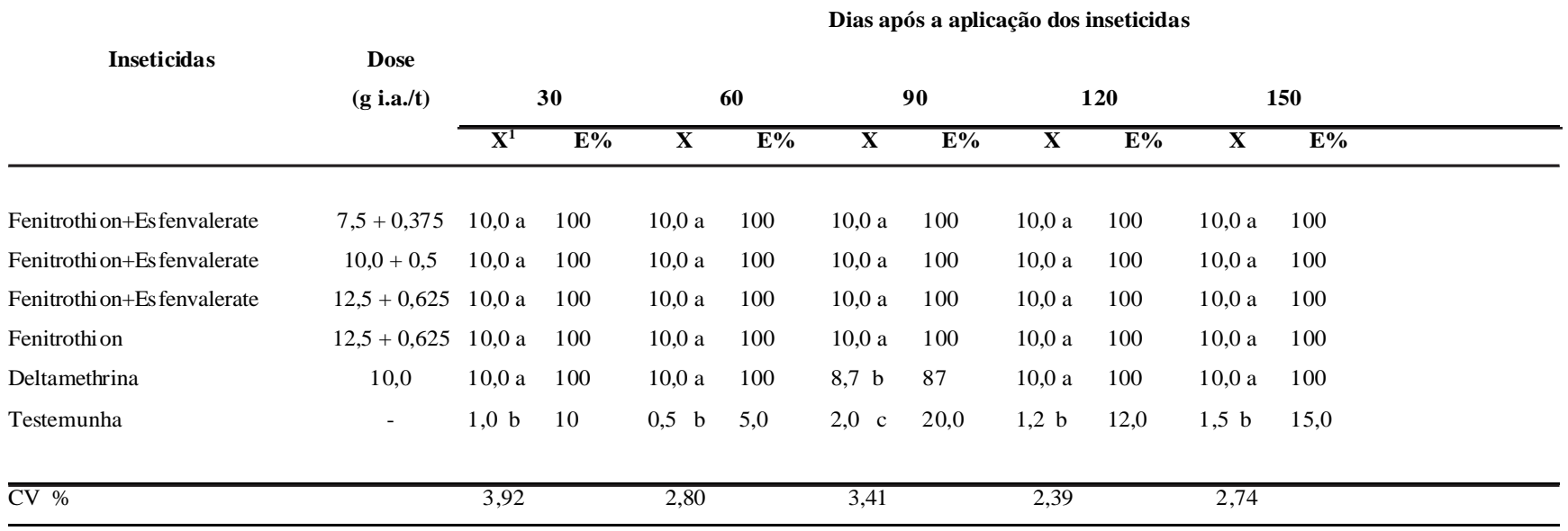

1. Médias seguidas pela mesma letra não diferem entre si pelo Teste de Tukey $(\mathrm{P}<0,05)$.

Tabela 2 - Número médio de adultos mortos $(\mathrm{X})$ de Rhyzopertha dominica e porcentagem de eficácia dos inseticidas (E\%) em diferentes períodos de infestação após o tratamento dos grãos de cevada.

Table 2 - Average number of dead adults (x) of Rhyzopertha dominica and percentage of insecticides effectiveness (E\%) in different periods of infestation after the treatment of the barley grains.

\begin{tabular}{|c|c|c|c|c|c|c|c|c|c|c|c|}
\hline \multirow{3}{*}{ Inseticidas } & \multirow{3}{*}{$\begin{array}{c}\text { Dose } \\
\text { ( g i.a./t) }\end{array}$} & \multicolumn{10}{|c|}{ Dias após a aplicação dos inseticidas } \\
\hline & & \multicolumn{2}{|c|}{30} & \multicolumn{2}{|c|}{60} & \multicolumn{2}{|c|}{90} & \multicolumn{2}{|c|}{120} & \multicolumn{2}{|c|}{150} \\
\hline & & $\mathrm{X}^{1}$ & $\mathrm{E} \%$ & $\mathrm{X}$ & $\mathrm{E} \%$ & $\mathrm{x}$ & $\mathrm{E} \%$ & $\mathrm{x}$ & $\mathrm{E} \%$ & $\mathrm{X}$ & $\mathrm{E} \%$ \\
\hline Fenitrothion+Esfenvalerate & $7,5+0,375$ & 10,0 a & 100 & $10,0 \mathrm{a}$ & 100 & $10,0 \mathrm{a}$ & 100 & $10,0 \mathrm{a}$ & 100 & $7,7 \quad b$ & 77 \\
\hline Fenitrothion+Esfenvalerate & $10,0+0,5$ & $10,0 \mathrm{a}$ & 100 & $10,0 \mathrm{a}$ & 100 & $10,0 \mathrm{a}$ & 100 & $10,0 \mathrm{a}$ & 100 & 10,0 a & 100 \\
\hline Fenitrothion+Esfenvalerate & $12,5+0,625$ & $10,0 \mathrm{a}$ & 100 & $10,0 \mathrm{a}$ & 100 & $10,0 \mathrm{a}$ & 100 & $10,0 \mathrm{a}$ & 100 & $10,0 \mathrm{a}$ & 100 \\
\hline Fenitrothion & $12,5+0,625$ & $7,0 \quad b$ & 70 & $7,5 \quad b$ & 75 & $5,2 \quad b$ & 52 & $6,7 \quad b$ & 67 & $5,0 \quad \mathrm{c}$ & 50 \\
\hline Deltamethrina & 10,0 & $9,7 \mathrm{a}$ & 97 & $10,0 \mathrm{a}$ & 100 & $10,0 \mathrm{a}$ & 100 & $10,0 \mathrm{a}$ & 100 & $9,7 \mathrm{a}$ & 97 \\
\hline Testemunha & - & $0,5 \mathrm{c}$ & 5 & $0,2 \mathrm{c}$ & 2 & $0,7 \quad \mathrm{c}$ & 70 & $1,0 \quad \mathrm{c}$ & 10 & $0,7 \mathrm{~d}$ & 7 \\
\hline $\mathrm{CV} \%$ & & 7,54 & & 7,46 & & 7,65 & & 5,88 & & 12,81 & \\
\hline
\end{tabular}

1. Médias seguidas pela mesma letra não diferem entre si pelo Teste de Tukey $(\mathrm{P}<0,05)$. 


\section{Referências}

ALMEIDA, A. A. 1989. Natureza dos danos causados por insetos em grãos armazenados. In: CONGRESSO BRASILEIRO DE ENTOMOLOGIA, 11, 1987, Campinas, SP, Anais..., Campinas: Fundação Cargill, 1987. v. 4, p. 16-32.

ABBOTT, W. S. A method of computing the effectiveness of na insecticide. J. Econ. Entomol, v. 18, p. 265-267, 1925.

BARETH, S. S.; GUPTA, H. C. Efficacy of gunny bag and seed treatment on the natural infestation of Rhyzopertha dominica on stored wheat. Seed Res. v. 17 , n. 2, p. 178-181, 1989a.

BARETH, S. S.; GUPTA, H. C. Efficacy of six insectides for the protection of stored wheat seeds against Rhyzoperta dominica (Fab.) Seed Res. v. 17, n. 1, p. 43-46, 1989b.

DUGUET, J.; QUAN, L Evaluation of the effectivness of deltamethrin spray or dust on rice husks against stored products pests on stored rice in southern of China. Agronomie Tropicale, v. 45, n. 2, p. 107113, 1990.

GIGA, D. P.; ZVOUTETE, P. The evaluation of different insecticides for the protection of maize against some stored product pests. I. Pest Cont. v. 32, n. 1, p. 10-13, 1990.

GUEDES, R. N. C.; SILVA, F. A. P. da. Avaliação do efeito residual do inseticida abamectin no controle de Sitophilus zeamaisMotschulsky (COLEOPTERA: CURCULIONIDAE) em milho armazenado. Rev. Ceres, v. 39, n. 225, p. 435-442, 1992.
ISLAM, N. et al. Comparative efficacy of different material against Sitophilusoryza e L. infesting maize seeds in storage. Bang. J. of Zool. v. 17, n. 2, p. 175-178, 1989.

LORINI, I. \& S. SCHNEIDER. Pragas de grãos armazenados: resultados de pesquisa. Passo Fundo, Embrapa - CNPT, 1994. p. 48.

PACHECO, I. A.; SARTORI, M. R.; YOKOMIZO, Y. Suscetibilidade de Sitophilusoryzae(COLEOPTERA: CURCULIONIDAE) ao piretróide deltametrina. Coletânea do Instituto de Tecnologia de Alimentos, v. 18, n. 1, p. 84-94, 1988.

SAMSO N, P. R.; PARKER, R. J.; HAL, E. A. Synergized deltamethrin as a protectant against Sitophilus zeamais Motsch. and S. oryzae (L.)(COLEOPTERA: CURCULONIDAE) on stored maize. J. Stored Prod. Res. v. 26, n. 3, p. 155-161, 1990.

SCHIFFERS, B. C. et al. The comparative toxicity of five pyrethroid inseticides to six insect pests of stored products. Medelingen van de Faculteit Landbouwwetenschappen, Rijksuniversiteit Gent, v. 54, n. 3b, p. 1095-1103, 1989.

SUCHITA, M. G.; REDDY, G. P. V.; MURTHY, M. M. $K$. Relative efficacy of pyretroids against rice weevil (Sitophilus oryzae L) infesting stored wheat. I. J. of Plant Prot. v. 17, n. 2, p. 243-246, 1989.

YADAV, T.D. Efficacy of deltamethrin againststorage insects in rice and wheat under FCI's storage system. Pest. v. 22, n. 12, p. 39-43, 1988. 